\title{
The effectiveness Study of Different Membranes in Treating Industrial Wastewater
}

\author{
Munawar Zaman Shahruddin, Izzah Sofiahanun Binti Ishak, Nur Hidayati Othman, Nur Hashimah Alias and Nur Azrini \\ Ramlee
}

Universiti Teknologi MARA, Malaysia

\begin{abstract}
This paper focuses on the effectiveness study of different membranes in treating petroleum based wastewater. Polysulfone (PSF) and Polyether Sulfone (PES) were prepared using phase inversion method in NMP solvent. The composition of membrane was varied by manipulating the PVP additives concentration $(5 \mathrm{wt} \%)$ in the membrane dope solution. The morphological study of prepared membranes was analysed using Field Emission Scanning Electron Microscope (FESEM) while the permeating flux and Chemical Oxygen Demand (COD) rejection rate were determined accordingly by using the refinery wastewater as the feed. The result found that the permeating flux increased as the operating pressure increased. However, for COD rejection, it was reported that only certain membrane show the best rejection. To clarify and support this finding, the membrane performance was further analyzed by determining the Total Dissolved Solid (TDS) rejection for PSF2 and PES2 membrane samples. The result showed that both membranes recorded relatively high TDS rejection which can be justified by the morphological studies of each samples. Therefore, this study proved that the membrane treatment have a high potential to treat the industrial wastewater for a larger scale.
\end{abstract}

\section{Introduction}

Ever since the establishment of the Petroleum Development Act (1974), it can be seen that development of oil and gas industry in Malaysia has been increasing year by year. This situation brings a lot of benefits and advantages in terms of country's profit, supporting a huge of job vacancies to local people and also gives a lot of investment opportunity to foreign and local investor.

However, this increasing industrialization has given some impact on ecosystem and environment which human relies on. This is because the wastewater from industry and refinery has become one of major source that contributes to water pollution.

Discharging industrial wastewater without being treated further may contaminate water and soil that eventually will result-in disturbances to the ecosystem. On initial discharge, these wastewaters may comprise high levels of inorganic pollutants which can be easily biodegradable, however Total Dissolved Solids (TDS), Biochemical Oxygen Demand (BOD) or Chemical Oxygen Demand (COD) can give bad impact on the ecosystems if their content in the waste water even maybe in tens of thousands $\mathrm{mg} / \mathrm{L}$.

The conventional wastewater treatment consists of a combination of physical, chemical and biological process and work to remove solids, organic matter and sometimes, nutrients from wastewater. It starts with the preliminary processes, followed by primary treatments which are focusing the physical separation of contaminants in the effluent. Next step would be secondary treatment which involves biological degradation of bacteria in order to decompose the organic pollutants in the effluent. The last stage of treatment would be tertiary treatment which is additional treatment if both of earlier treatments cannot achieve the standard or requirement as per requested.

In a conventional wastewater treatment plant operation, three major process mainly involve: biochemical treatment, liquid/solid separation operation for sludge treatment and valorization [1]. In scope of petroleum industry, there are two basic treatment stage involve where are mechanical and physicochemical treatment in the first stage and followed by the advanced treatment of the pre-treated primary effluent [2].

Apart of that, there are other several methods that been proposed in treating industry wastewater such as electrochemical coagulation [3], electron Fenton [4], membrane bioreactor [5], photocatalytic reaction [6], catalytic ozonation [7] and membrane [8]-[10].

A lot of study on membrane technology has been conducted as the researchers finds that membrane could be a good option to treat and improve the quality of wastewater. According to Saljoughi \& Mousavi [10], membrane processes have been regarded as an advanced treatment compared to conventional wastewater treatment processes. The benefits of advanced treatment compared to conventional process are due to its characteristic such 
as selective preparation, continuous and automatic operation, and easy and well-arranged process conduction.

There are some researches done by several experts on the application of microfiltration or ultrafiltration membrane in industrial wastewater treatment. A review on the applicability of integrated/hybrid membrane processes in water treatment and reverse osmosis desalination plants have been studied where the result founds that it has huge potential to be widely applied and can lead to breakthrough in solving the problem of water scarcity [11].

Salahi et al. [12] reported the performance evaluation of five different polymeric membranes including two ultrafiltration and three ultrafiltration for treatment of an industrial oily wastewater, where the result found that ultrafiltration membrane resulted-in highest flux and higher rejection. Lin \& Lan [8] investigated the treatment of waste drawing oil which is commonly used in the cable and wire industries by employing semi batch ultrafiltration (UF) and reverse osmosis (RO). The result showed that the water quality of permeated from combine processes displayed excellent performance. Salahi et al. [13] tested the asymmetric Polyether Sulfone ultrafiltration membrane for oil wastewater treatment and the result reported that Polyether Sulfone is effective in removing COD, turbidity etc.

Chakrabarty et al. [9] in their study found that morphological properties of membrane due to addition of PVP and PEG have a significant influence on the permeate flow rate and oil removal. This will lead to the emergence of the wastewater treatment by using the polymeric membranes.

\section{Methodology}

\subsection{Materials}

The main polymers used in this study are PSF and PES with having the same average weight of $35000 \mathrm{Da}$ were provided by BASF. Reagents grade N-methyl-2pyrrolidone (NMP) (99.5\% purity) used as solvent were purchased from Anachemia Ltd. Reagent grade Polyvinyl pyrrolidone (PVP) (average molecular $58000 \mathrm{Da}$ ) was used as additive in casting solution in order to get membrane with varying morphological and permeation properties. Distilled water was used as main non solvent in the coagulation bath in preparing the membrane. Wastewater sample was collected from the refinery plant.

Table 1. Membrane dope composition

\begin{tabular}{|c|c|c|}
\hline $\begin{array}{c}\text { Polymer/Membrane } \\
(\mathbf{2 0} \mathbf{w t} \%)\end{array}$ & $\begin{array}{c}\text { PVP } \\
\text { (wt\%) }\end{array}$ & $\begin{array}{c}\text { NMP } \\
\text { (wt \%) }\end{array}$ \\
\hline PSF1 & 0 & 80 \\
\hline PSF2 & 5 & 75 \\
\hline PES1 & 0 & 80 \\
\hline PES2 & 5 & 75 \\
\hline
\end{tabular}

PSF and PES beads were dried in oven for 1 hour at $80{ }^{\circ} \mathrm{C}$ before being used. Phase inversion method was used to synthesis the flat sheet membrane by using distilled water as non-solvent. Both of the polymers were dissolved separately in the solvent of NMP at room temperature $\left(25^{\circ} \mathrm{C}\right)$. After the polymer was completely dissolved in NMP solution, then PVP reagent was added. Table 1 represent the composition of different membranes. The PSF and PES concentration was kept constant at $20 \%$, keeping the solvent and additive concentration at $80 \%$. Each dope solution needs to be mixed properly by stirring the solution at $200 \mathrm{rpm}$. Next, the prepared solution was casting manually using casting knife with the thickness of $150 \mu \mathrm{m}$. The synthesized membrane was then immersed in distilled water for 24 hours. Then the formed membrane was dried at room temperature for one day. The synthesized membranes were designated as PSF1, PSF2, PES1 and PES2.

\subsection{Membrane characterization}

\subsubsection{Permeating flux and rejection rate}

In this study, permeating flux, $\mathrm{J}\left(\mathrm{m}^{3} / \mathrm{m}^{2} . \mathrm{s}\right)$ for each membrane was determined at different pressure $(2$ and 4 Bar) using wastewater as the feed by employing the following equation:

$$
\mathrm{J}=\mathrm{Q} /(\text { A.t) }
$$

where $Q$ is the volume of permeate $\left(\mathrm{m}^{3}\right)$, A is the effective membrane area $\left(\mathrm{m}^{2}\right)$ and $\mathrm{t}$ is the time obtain the volume of Q (s).

To assess the separation efficiency of each membrane, the rejection rate, $\mathrm{R}(\%)$ is used and can be calculated using the following formula:

$$
\mathrm{R}=\left(1-\mathrm{C}_{\mathrm{f}} / \mathrm{C}_{\mathrm{p}}\right) \times 100
$$

where $C_{f}$ and $C_{p}$ are the concentration (any parameter of interest) of feed and membrane permeate. The membrane setup can be found in Fig. 1.

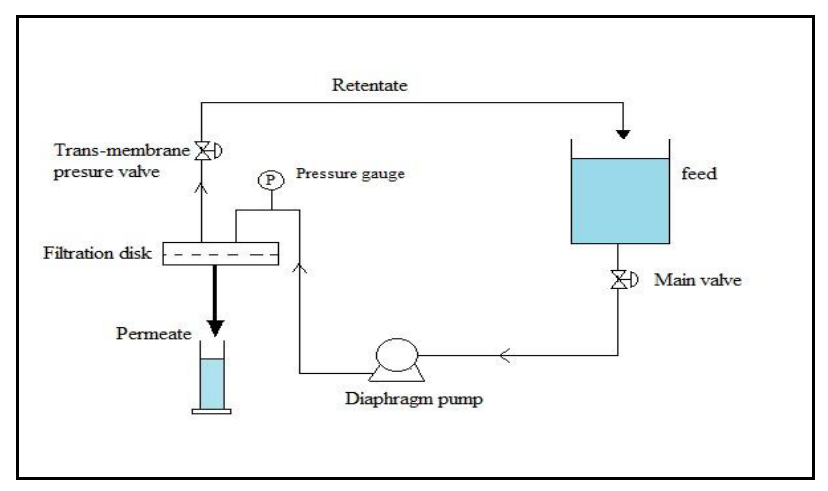

Figure 1. Membrane permeation testing rig

2.3.2 Analytical COD and TDS 
Analysis on the COD concentration of the feed and permeate sample were conducted using Hach Spechtrophotometer (Model DR2800). Meanwhile TDS meter (Model Hach) was used to determine the concentration of TDS in feed and permeate.

\subsubsection{Morphological study}

The prepared membranes were characterized by morphological analysis and permeation experiments. The morphological of the prepared membrane was studied using FESEM. The membrane sample was dried and then fractured cryogenically in liquid nitrogen before mounting on sample stud. The samples were then sputtered with a thin layer of gold using a sputtering apparatus. Various FESEM images were taken at magnification of 1500 for cross sectional views.

\section{Results and discussion}

The refinery wastewater was characterized in terms of several water quality parameters as mentioned in the methodology before being treated. The value for all parameters can be seen in Table 2 .

Table 2. Refinery wastewater sample characteristics

\begin{tabular}{|c|c|}
\hline Wastewater Characteristic & Value \\
\hline Conductivity & $3.38 \mathrm{mS} / \mathrm{cm}$ \\
\hline Total Dissolved Solid (TDS) & $1758 \mathrm{mg} / \mathrm{L}$ \\
\hline Chemical Oxygen Demand (COD) & $153 \mathrm{mg} / \mathrm{L}$ \\
\hline
\end{tabular}

\subsection{Membrane permeating flux}

Fig. 2 shows the trend of permeating flux with two different operating pressures for four different types of membrane.

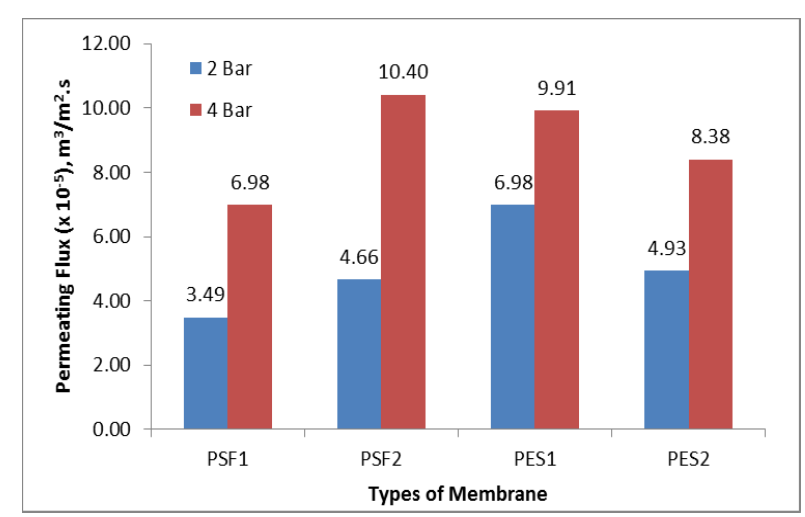

Figure 2. Permeating flux for all membrane samples

As can be seen in the figure, the trend shows that the flux increase linearly with the operating pressure for all membrane sample. Specifically, for PSF membrane (PSF1 and PSF2), the graph shows that membrane fluxes for PSF2 is higher than PSF1. This shows that the addition of PVP additive affect the membrane performance by increasing the flux value, hence making the membrane performance become well. This result is coherent with the study done by Pagidi et al.[14], where their finding shows that all the blended membrane exhibited a higher permeation flux than the pure membrane. For PES, the graph also show the same trends where both of PES1 and PES2 have increasing flux when operating pressure increase. In term of additive addition into PES membrane solution, the trend recorded viceversa as to compare with PSF membrane. This phenomenon is resulted from the non-uniform distribution of micropores as a result of the addition of PVP in the membrane dope solution. Ismail \& Hassan [15] also found the same finding in their study. Plus, this result could be strongly supported with Barth et al.[16] where their study also shows the same result.

From the graph, a comparison for PSF and PES membrane shows that the fluxes are higher for PSF membrane. The reason for this finding could be credited to the thick sponge-like support layer of the PSF membrane which additionally permits the flux of bigger molecules. Besides, the different polarities of membrane material also could affect the membrane fluxes [16].

\subsection{Rejection rate}

\subsubsection{COD rejection rate}

The performance of membrane sample further analyzed by estimating the rejection during permeation experiment with wastewater as feed. For this purpose, the wastewater quality chosen is COD as the indicator to observe the quality of wastewater before and after membrane filtration.

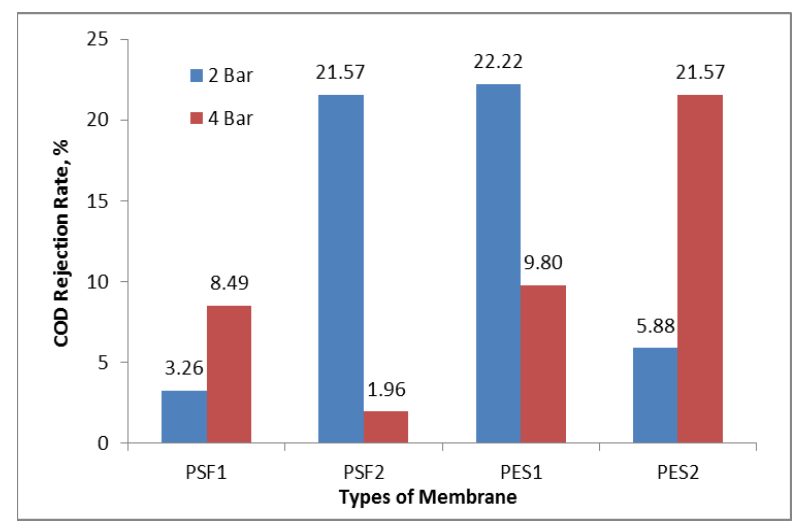

Figure 3. COD rejection rate for all membrane samples

Fig. 3 shows the trends of COD rejection for PSF1, PSF2, PES1 and PES2 membrane sample. As for PSF1, it can obviously be seen that as the operating pressure increased, the COD rejection also increased. This is because as the operating pressure increase, the flux also increase and the amount of retained organic material that contained in wastewater, attached on membrane surface also increase. So the increase of the operating pressure make it possible to decrease the COD concentration in permeate. 
While for PES1, the trend shows that COD rejection decline with the increasing operating pressure. If referring to membrane flux (Fig. 2) for PES1, it exhibits that the membrane flux increase with the operating pressure, so theoretically, the COD rejection also should incline with the operating pressure. However, it happen vice versa. This same situation happen to Zheng et al.[17] where their research also show the same result. It can be concluded that the increase of permeate flux, the increase of the amount of the retained organic material across the membrane, thereby resulting an increase in COD concentration in permeate and reduce the COD rejection rates.

As for PSF1 and PSF2, their rejection performance can be compared in terms of additive contribution. As mentioned before, PSF1 is pure polysulfone membrane while PSF2 is the blend membrane with $5 \mathrm{wt} \%$ PVP. For comparison, as the operating pressure increase, COD rejection rate for PSF1 increasing, while for PSF2 it is recorded there is declining in COD rejection rate. This is because the roles of PVP additives in polymer solution as a micropores promoter and increase the porosity and supressed the macrovoids formation. So in this study, it can be concluded that as the PVP additives is added in the solution, the pore area per unit surface area (porosity) increase, hence the permeate flux also increase and thereby the COD rejection is decline [18, 19]. This is because as bigger the pore area, more organic material can pass through the membrane.

While for both PES1 and PES2, Fig. 3 shows different trends of COD rejection. As the operating pressure increase, the COD rejection for PES1 decreasing, whereas PES2 show increasing trends. Theoretically, as additive is added, the membrane pore radius increase. Hence, as the operating pressure increase, so the membrane flux also increase, hence causing the rejection rate decrease. The result for PES2 logical accordingly with Ismail \& Hassan [15] research.

\subsubsection{TDS rejection rate}

The performance of membrane is further analyzed with reference of rejection of Total Dissolved Solid (TDS) in the permeate.

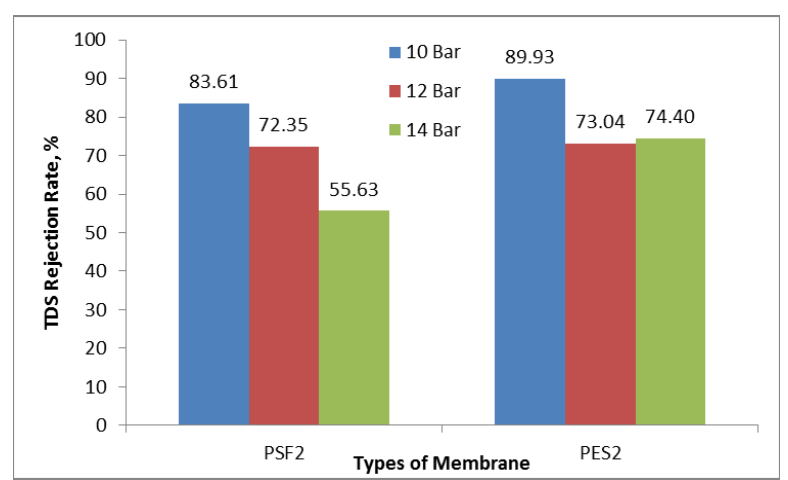

Figure 4. TDS rejection rate for the membrane with the addition of PVP

This analysis is done to clarify and support the result obtained earlier on COD rejection. For this purpose, membrane PSF2 and PES2 are chosen in the analysis in order to study the significance of PVP additive in membrane performance in term of TDS rejection.

For PES2 it is observed that as the operating pressure increase, the rejection of TDS decrease but at 14 bar, it increase slightly from $73 \%$ to $74 \%$. This trend does not follow the theory. This situation could arise due to the compaction factor, where it is related to the structure of the membrane especially membrane sub layer. The higher compaction factor, the more likely membrane compact because of the existence of large number of macro voids in the sub layer resulting in narrower passage for permeate flow. Generally, membrane with bigger pore size combined with loose structure result in poor separation performance [9].

\subsection{Membrane morphological study}

From Fig. 5 it can be seen that membrane formed are having asymmetric structure layer consisting of a dense top layer and a porous sublayer. For comparison, the macropores for PSF2 is smaller compare to PSF1. This is truly true with the fact that PVP additives function to suppress the macropores (Han \& Nam, 2002). Therefore the flux data, showing correlation with PVP content in membrane.
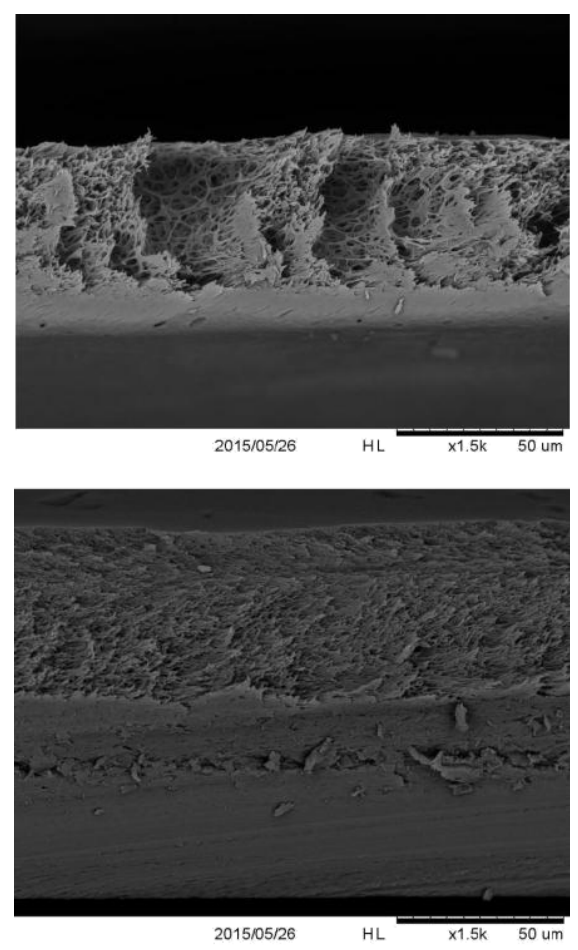

Figure 5. FESEM images of cross sectional for PSF1 (below) and PSF2 (above) membrane sample

As shown in Fig. 6, both PES1 and PES2 have asymmetric structure consists of a dense top layer and finger like porous structure. The cross section image revealed that PES2 have thicker effective skin layer with larger pore size compare to PES1, thus exhibiting low permeating flux and higher rejection rate. This finding 
correlates with the permeability performance of both membrane as being discussed previous.
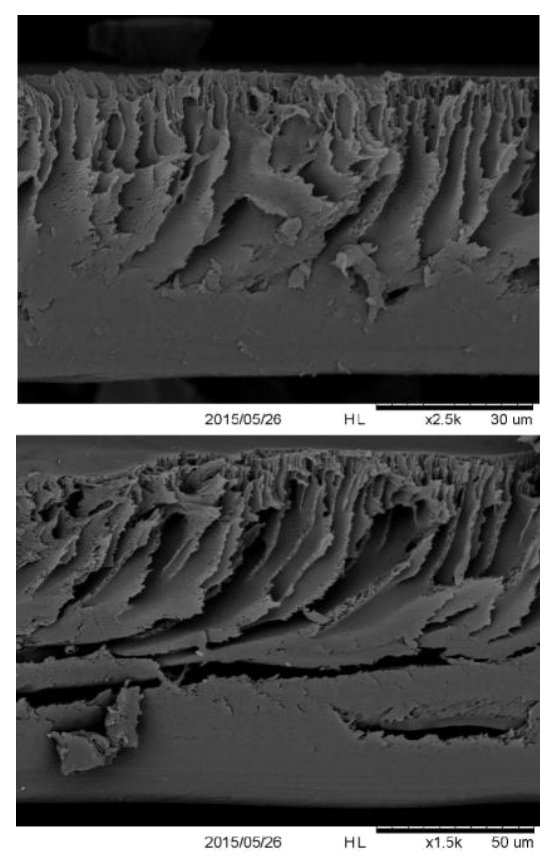

Figure 6. SEM images of cross sectional for PES1 (above) and PES2 (below) membrane sample.

\section{Conclusion}

The objective of this study is to develop the effectiveness of two different membranes consists of PSF and PES, by manipulating the additive concentration in dope solution to treat the wastewater. Then, the performances of membranes prepared were characterized in terms of permeating flux, COD and TDS rejection and membrane morphological. The following conclusion can be drawn from the study:

1. Membrane have been successfully fabricated using phase inversion method using PSF and PES as the main polymer, NMP as the solvent and PVP as the additive.

2. Membrane testing using wastewater as the feed shows that, as the operating pressure increase, the performance of membrane increase. Besides, the addition of PVP additives also enhances the membrane performance for certain membrane sample. However, there is other parameter that should be taking into account such as the thickness of membrane, the surface membrane chemistry, size of organic matter, fouling etc. which could affect the performance of membrane.

3. The percentage of COD removal in this study show low rejection while the percentage of TDS removal shows high rejection.

4. The SEM images show the structural illustration of membrane $n$ term of cross section and top surface. By analyzing this data, it helps in improving the understanding on how the membranes perform.
This work is supported via financial fund namely the Fundamental Research Grants Scheme (FRGS): 600RMI/FRGS 5/3 (75/2015) managed by Research Management Centre, Universiti Teknologi MARA, Shah Alam, Malaysia.

\section{References}

1. N. Descoins, S. Deleris, R. Lestienne, E. Trouvé, and F. Maréchal, "Energy efficiency in waste water treatments plants: Optimization of activated sludge process coupled with anaerobic digestion," Energy, vol. 41, no. 1, pp. 153-164, 2012.

2. B. H. Diya'Uddeen, W. M. A. W. Daud, and a. R. Abdul Aziz, "Treatment technologies for petroleum refinery effluents: A review," Process Saf. Environ. Prot., vol. 89, no. 2, pp. 95-105, 2011.

3. C. L. Yang, "Electrochemical coagulation for oily water demulsification," Sep. Purif. Technol., vol. 54, no. 3, pp. 388-395, 2007.

4. R. Davarnejad, M. Mohammadi, and A. F. Ismail, "Petrochemical wastewater treatment by electroFenton process using aluminum and iron electrodes: Statistical comparison," J. Water Process Eng., vol. 3, pp. 18-25, 2014.

5. M. M. Rahman and M. H. Al-Malack, "Performance of a crossflow membrane bioreactor (CF-MBR) when treating refinery wastewater," Desalination, vol. 191, no. 1-3, pp. 16-26, 2006.

6. F. Shahrezaei, Y. Mansouri, A. A. L. Zinatizadeh, and A. Akhbari, "Process modeling and kinetic evaluation of petroleum refinery wastewater treatment in a photocatalytic reactor using $\mathrm{TiO} 2$ nanoparticles," Powder Technol., vol. 221, pp. 203212, 2012.

7. C. Chen, L. Wei, X. Guo, S. Guo, and G. Yan, "Investigation of heavy oil refinery wastewater treatment by integrated ozone and activated carbon supported manganese oxides," Fuel Process. Technol., vol. 124, pp. 165-173, 2014.

8. S. H. Lin and W. J. Lan, "Waste oil/water emulsion treatment by membrane processes," J. Hazard. Mater., vol. 59, no. 2-3, pp. 189-199, 1998.

9. B. Chakrabarty, a. K. Ghoshal, and M. K. Purkait, "Ultrafiltration of stable oil-in-water emulsion by polysulfone membrane," J. Memb. Sci., vol. 325, pp. 427-437, 2008.

10. E. Saljoughi and S. M. Mousavi, "Preparation and characterization of novel polysulfone nanofiltration membranes for removal of cadmium from contaminated water," Sep. Purif. Technol., vol. 90, pp. 22-30, 2012.

11. W. L. Ang, A. W. Mohammad, N. Hilal, and C. P. Leo, "A review on the applicability of integrated/hybrid membrane processes in water treatment and desalination plants," Desalination, 2014.

12. A. Salahi, A. Gheshlaghi, T. Mohammadi, and S. S. Madaeni, "Experimental performance evaluation of polymeric membranes for treatment of an industrial

\section{Acknowledgement}


oily wastewater," Desalination, vol. 262, no. 1-3, pp. 235-242, 2010.

13. A. Salahi, T. Mohammadi, and R. Mosayebi, "Journal of Environmental Chemical Engineering Asymmetric polyethersulfone ultra fi ltration membranes for oily wastewater treatment: Synthesis, characterization , ANFIS modeling, and performance," Biochem. Pharmacol., vol. 3, no. 1, pp. 170-178, 2015.

14. A. Pagidi, R. Saranya, G. Arthanareeswaran, a. F. Ismail, and T. Matsuura, "Enhanced oil-water separation using polysulfone membranes modified with polymeric additives," Desalination, vol. 344, pp. 280-288, 2014.

15. A. F. Ismail and A. R. Hassan, "Effect of additive contents on the performances and structural properties of asymmetric polyethersulfone (PES) nanofiltration membranes," Sep. Purif. Technol., vol. 55, no. 1, pp. 98-109, 2007.

16. C. Barth, M. C. Gonçalves, a. T. N. Pires, J. Roeder, and B. a. Wolf, "Asymmetric polysulfone and polyethersulfone membranes: Effects of thermodynamic conditions during formation on their performance," J. Memb. Sci., vol. 169, no. 2, pp. 287-299, 2000.

17. Y. Zheng, S. Yu, S. Shuai, Q. Zhou, Q. Cheng, M. Liu, and C. Gao, "Color removal and COD reduction of biologically treated textile effluent through submerged filtration using hollow fiber nanofiltration membrane," Desalination, vol. 314, pp. 89-95, 2013.

18. B. Chakrabarty, a. K. Ghoshal, and M. K. Purkait, "Preparation, characterization and performance studies of polysulfone membranes using PVP as an additive," J. Memb. Sci., vol. 315, no. 1-2, pp. 3647, 2008.

19. M. J. Han and S. T. Nam, "Thermodynamic and rheological variation in polysulfone solution by PVP and its effect in the preparation of phase inversion membrane," J. Memb. Sci., vol. 202, no. 1-2, pp. 55-61, 2002. 